\title{
Instruction Level Duplication and Comparison for Data Error Detection: a First Experiment
}

\author{
Venu Babu Thati ${ }^{1}$, Jens Vankeirsbilck ${ }^{1}$, Davy Pissoort ${ }^{2}$ and Jeroen Boydens ${ }^{1}$ \\ ${ }^{1}$ Department of Computer Science, M-Group, Campus Bruges, KU Leuven \\ ${ }^{2}$ Department of Electrical Engineering, M-Group, Campus Bruges, KU Leuven \\ Spoorwegstraat 12, 8200 Bruges, Belgium \\ \{VenuBabu.Thati | Jens.Vankeirsbilck | Davy.Pissoort| Jeroen.Boydens\}@kuleuven.be
}

\begin{abstract}
In this paper, we present a new software approach Instruction Level Duplication and Comparison (ILDC) for data error detection. We implemented ILDC in five different case studies. To validate our proposed technique, we measured its fault detection ratio and execution time overhead. Next, we compare it to the following two existing techniques: overhead reduction (VAR3+) and software-implemented fault tolerance (SWIFT), respectively. The results show that ILDC detects more errors than VAR3+ and SWIFT at a lower overhead.
\end{abstract}

Keywords - Embedded Systems, Fault Tolerance, Data Flow Errors, Duplication and Comparison

\section{INTRODUCTION}

In recent years, new approaches for embedded systems have been emerging and have become more susceptible to erroneous bit-flips. This is due to a reduction in the size of components and supply voltages and due to increasing complexity levels of the latest computer architectures [1]. More specifically, such erroneous bit-flips, called soft errors, are caused by factors including drastic changes in environments such as temperature fluctuations [2], electromagnetic interference [3], background radiation and power supply ripple voltage. Soft errors play a major role in embedded systems and can cause unusual behavior by degrading the system's integrity and reliability [4].

In order to improve the reliability and integrity of systems, fault tolerance should be included. This allows detecting an error and recovering it. In recent years, fault tolerance has been mainly achieved through hardware-based solutions. Such solutions are based on redundancy techniques that meet the requirements of reliability and integrity. However, hardware redundancy is a relatively costly process, because of the extra hardware per product [4].

To reduce the extra cost and overhead per product, alternative techniques based on software redundancy have been proposed and used. These techniques do not need an extra hardware for their implementation. Because of this, the cost is minimized. In software-based techniques, errors can be detected by including extra code into the program without changing the corresponding hardware [5]. These techniques require extra processing time and memory due to the execution of the extra code. Because of their cost and flexibility, these software-based solutions are used in numerous applications [6].

According to recent studies, soft errors are one of the immediate sources of failure in embedded systems [4,7]. Such soft errors can have several effects on embedded systems. Since more systems are in use and the environment becomes harsher, a system crash or failure is more likely to occur. From a software perspective, the effects of soft errors can be divided into the two categories: data flow and control flow errors. A data flow error can lead to a corrupted intermediate or final output of the program. In contrast, a control flow error can lead to jumps in the program execution order, causing a program hang or crash [8].

This paper focuses on data flow errors. An overview of control flow error detection techniques is given by Vankeirsbilck et al. [8]. To counter data flow errors, numerous techniques have been proposed and implemented for several years [9]. In this paper, we propose a new data error detection technique; instruction level duplication and comparison (ILDC). ILDC aims to improve the fault detection ratio without having much impact on programs execution time overhead. This is achieved by duplicating all the instructions in the program except branch instructions and placing comparison instructions in selective places.

The remainder of the paper is organized as follows. Section II describes the related work in the literature. Section III discusses the newly proposed technique. Section IV discusses the experimental setup. Section V presents the results obtained through experiments. Section VI concludes this paper and our future work plans are given in Section VII.

\section{RELATED WORK}

As aforementioned, numerous techniques for data flow error detection based on duplication and comparison exist. To evaluate the proposed technique, we considered two existing detection techniques based on their similarities with our technique; overhead reduction (VAR3+) and software-implemented fault tolerance (SWIFT). In VAR3+, all instructions in the entire code base, except store and branch instructions are duplicated. The main idea of not duplicating store instruction is to avoid creating multiple memory locations, which leads to a reduction in overheads. Next, comparison instructions are placed before load, store and branch instructions to compare the results [5].

Another considered technique is referred to as SWIFT. In this technique, all instructions except store instructions are duplicated [10]. The idea of bypassing store instructions from duplication is to avoid multiple memory locations. Comparison instructions are placed before store and branch instructions to compare the results and report errors. In this way, the considered techniques are succeeded in improving their overheads. 
Despite the lower overheads in the considered techniques, the main drawback is a reduction in fault detection as can be seen in [11]. This is due to the position of their comparison instructions to detect errors. In this paper, our technique aims to overcome the identified drawbacks. This can be achieved by placing comparison instructions in the correct position to detect more errors.

\section{ILDC TECHNIQUE}

The general idea involved in data flow error detection techniques is to duplicate instructions. As a consequence, duplicate registers are needed. By duplicating the registers, it is possible to detect data flow errors by comparing original and duplicated registers.

In order to detect data flow errors, the current ILDC consists of the following three key steps:

1) Every register has a duplicate.

2) All instructions, except branch instructions, are duplicated using the duplicate registers.

3) Comparison instructions are inserted at selective places:

$\begin{array}{cl}\text { i. } & \text { Before store instructions } \\ \text { ii. } & \text { Before branch instructions } \\ \text { iii. } & \text { After load instructions } \\ \text { iv. } & \text { After move instructions. }\end{array}$

The first step regards how the registers are duplicated in the program. This can be done by using the unused registers. The second one relates to how the instructions are duplicated in the considered program. Here, all instructions except branch instructions need to be duplicated by using the duplicate registers obtained in the previous step. The main reason for not duplicating the branch instructions is that they could lead to an infinite loop or program hang. The third step refers to how and where the comparison instructions are inserted. These instructions are used to check if an error has occurred. If the results from the original and duplicated operation are different, an error is reported.

As aforementioned, in our ILDC, we insert comparison instructions in the following four positions:

i. Before store instructions: a comparison instruction is inserted before store operation. The core idea behind placing a comparison immediately before storing register values in memory is to make sure the information to be stored is correct. In this way, more errors can be detected.

ii. Before branch instructions: a comparison instruction is inserted before branch instruction. The intuitive idea to place a comparison instruction before deciding the direction of a branch instruction is to check and provide correct information for further calculations.

iii. After load instructions: comparisons take place after loading the information from two different memory locations. In this case, comparisons are necessary after original and duplicate load information, since the loaded information is from two distinct locations.

iv. After move instructions: the final comparison rule is to place comparison instructions after the original and duplicate move operation. It is known from ARM Cortex-M architecture that the move is a data movement instruction, which loads the data from one register to the other. To ensure and report the data corruption during data transfer between registers, we placed comparison instructions after original and duplicated operations.

Table 1 shows an example of assembly-level unprotected code that is protected by different data error detection techniques: ILDC, VAR3+ and SWIFT. The unprotected code, shown in the first column, uses four registers: $r 0$ to $\mathrm{r} 3$. This means that the protected code, shown in the second, third and fourth columns have to use eight registers, one duplicate for each original register. The duplicates are indicated with the '-symbol. In addition, comparison instructions have to be placed based on the mechanism involved in the corresponding techniques to validate the results.

TABle 1. EXAmple For Assembly-LeVel IMPLEMENTEd DAtA ERror DETECTION TECHNIQUES

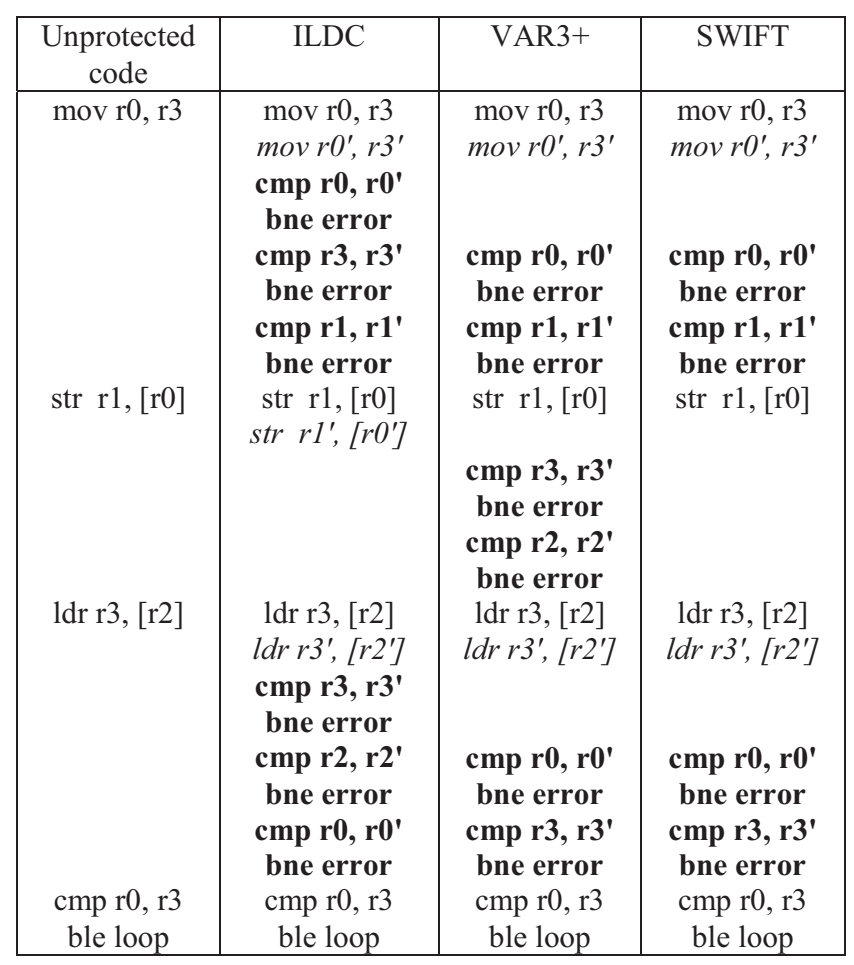

\section{EXPERIMENTAL SETUP}

This section presents the required components used for our experiments. We first start with describing which case studies were considered. Next, we present the hardware and fault injection process that were used for experiments.

\section{A. Case studies}

We selected five different case studies to implement and test our technique. These case studies are an implementation of the bubble sort (BS), matrix multiplication (MM), bit count $(\mathrm{BC})$, cyclic redundancy check $(\mathrm{CRC})$ and selection sort (SS) algorithms. Among them, BC and $\mathrm{CRC}$ are from the MiBench suite [6]. Being highly used as validation case studies in the published literature for the data error detection techniques, these case studies also have varying complexity and CFGs as shown by Vankeirsbilck et al. [8]. This makes them highly suitable for our experiments. 


\section{B. Hardware}

The selected case studies were executed on a NXP LPC1768, an ARM Cortex-M3 driven microcontroller. The ARM Cortex-M3 was selected because it is an industry leading 32-bit processor used in various embedded application domains [12].

\section{Fault injection process and evaluation}

To test our implementations, data errors need to occur in the target system. To make them occur, we inject bit-flips using our in-house fault injection tool. Since most systems already have some ECC countermeasures in place for their memory, we only inject bit-flips in CPU registers. To deterministically and directly inject data errors into the processor registers, the instruction aware injection mechanism was used $[11,13]$.

Further, we have integrated instruction aware injection mechanism into our in-house developed software-implemented fault injection (SWIFI) tool. This tool was also used to validate the implemented techniques in our previous publications [8,11]. Fig. 1 gives an architectural overview of the SWIFI tool. The external host PC is connected to the Cortex microcontroller software interface standard debug access port (CMSIS-DAP) interface of the processor to inject bitflips into the target hardware. The CMSIS-DAP interface allows us to remotely manipulate the data present in CPU registers using the on-chip debugger. Next, we injected 3200 data errors per case study for validation.

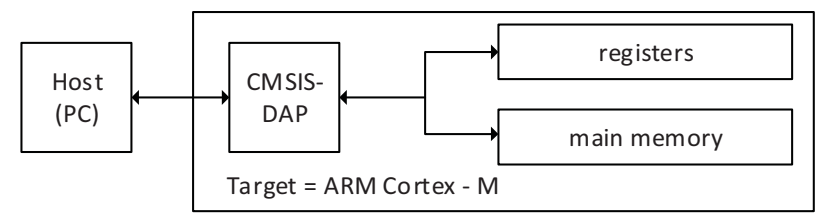

Fig. 1. The architecture of SWIFI tool

After validating fault injection, obtained results are analyzed based on the following classification:

Software Detected Fault (SDF): The injected fault is detected by the duplication and comparison instructions used for data error detection.
Hardware Detected Fault (HDF): the default hardware fault exception handler in ARM Cortex processor family detects the injected fault.

Silent Data Corruption (SDC): The injected fault is not detected and it changes the output of the program.

No Effect Fault (NEF): The injected fault does not have an effect on the output of the program.

\section{RESULTS COMPARISON AND DISCUSSION}

This section discusses the results achieved from our experiments. To evaluate our ILDC, we compare it with VAR3+ and SWIFT. This comparison is based on the four defined fault categories: SDF, HDF, SDC and NEF. On the other hand, we also make a comparison based on imposed execution time overhead.

Fig. 2 displays the results obtained from fault injection validation. The achieved results are in the form of four fault categories: SDF, HDF, SDC and NEF. Examining Fig. 2, the most interesting fault category for comparison is one of the faults detected by our ILDC technique in the form of SDF. It is clear from the first fault category that ILDC displays the highest percentage of SDF for all considered case studies. In comparison to VAR3+ and SWIFT, the average percentage of SDF in ILDC has improved by $37.5 \%$ and $42.5 \%$, respectively. In addition to the SDF, the percentage of faults detected in the HDF category is much lower among all case studies using three different techniques. In this category, faults are not detected by our ILDC but detected by the default hardware fault exception handlers. That is why this category is less important in our comparison.

The third fault category is referred to as SDC. It represents the faults that were not detected by the implemented data error detection technique. In this case, our ILDC shows the lowest percentage of undetected faults. More specifically, on average ILDC has $15.7 \%$ of SDC, whereas VAR3+ and SWIFT have $23.6 \%$ and $25.6 \%$, respectively. The final fault category is referred to as NEF. This category indicates the faults which do not have an effect on the output of the program. Therefore, this category cannot be considered for the comparison.

Erroneous bit-flip detection does not come cheap. They do impose extra execution time. Execution time overhead (ETO) is determined by the number of extra assembly

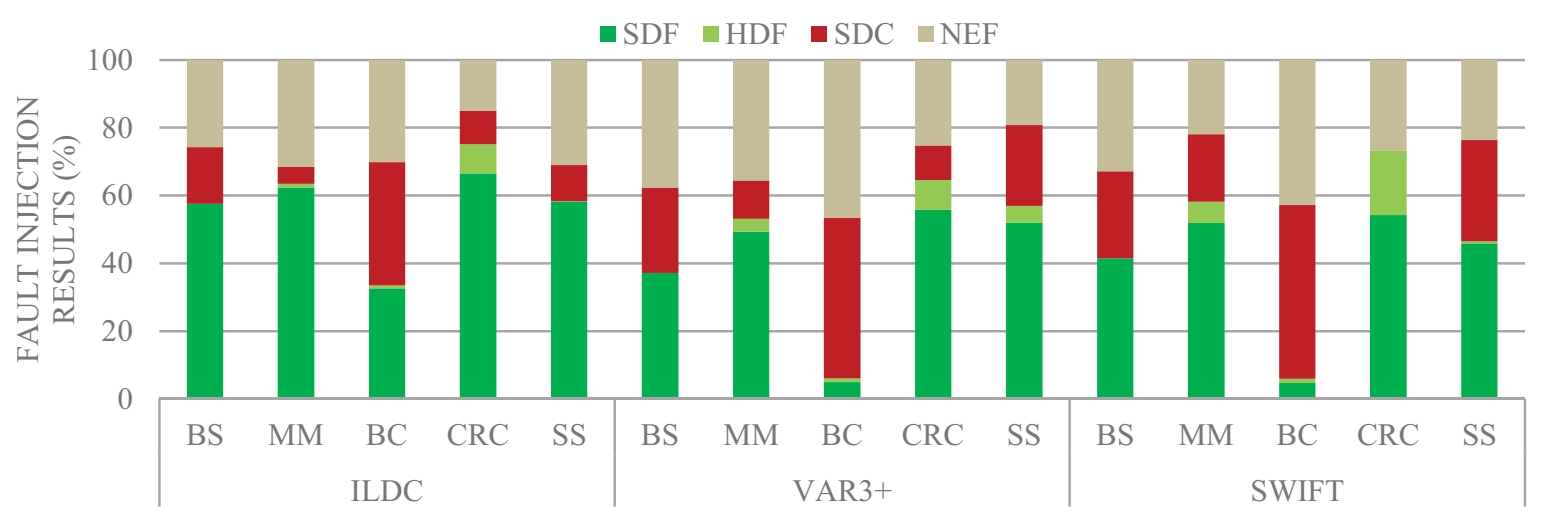

Fig. 2. Fault injection results of ILDC, VAR3+ and SWIFT 


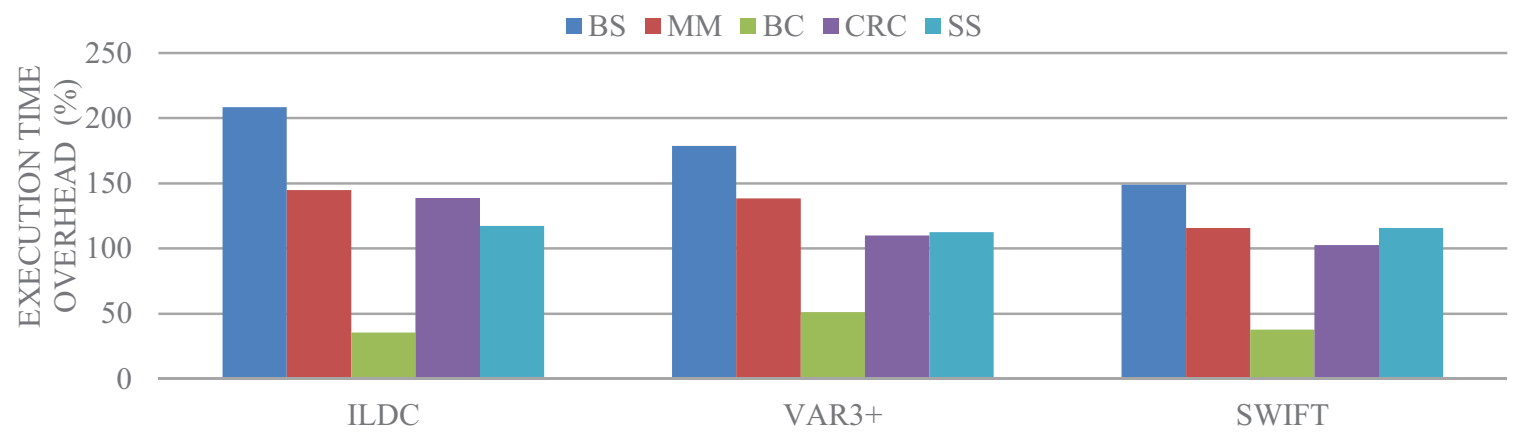

Fig. 3. Execution time overhead of ILDC, VAR3+ and SWIFT

instructions added to the program. Most of the times, it also depends on the type of instructions. In this study, the introduced ETO is calculated based on the following two parameters; protected execution time (PET) and unprotected execution time (UPET) as given in Eq. 1

$$
\text { ETO }=\quad \frac{P E T-U P E T}{U P E T} .
$$

Fig. 3 presents the overhead costs per technique per case study. Evaluating Fig. 3, it is clear that overheads in VAR3+ and SWIFT nearly have the same lower percentage for most case studies. On the other hand, the average execution time overhead of ILDC has increased by $16.5 \%$ in comparison to the average of VAR3+ and SWIFT, respectively. This is acceptable in comparison with the improvement that is achieved in fault detection.

\section{CONCLUSION}

In this paper, we presented a new technique for data flow error detection based on instruction level duplication and comparison. ILDC was implemented in five different case studies. We performed experiments for the five considered case studies. The performed experiments measured two criteria, namely fault detection and execution time overhead. The two criteria were then used to evaluate our technique by comparing it with VAR3+ and SWIFT techniques.

The first experimental results showed that ILDC detected the most data flow errors for all considered case studies. In comparison to VAR3+ and SWIFT, the percentage of software detected faults (SDF) in our ILDC has improved by $37.5 \%$ and $42.5 \%$, respectively. On the other hand, the execution time overhead has reduced slightly which is acceptable in comparison with the improvements that achieved in SDF.

\section{FUTURE WORK}

In this study, as a first experiment, we only considered one possible combination of comparison instructions. Moreover, our method was validated by using only five case studies. Although the obtained results with ILDC are promising in comparison with the VAR3+ and SWIFT, we would like to perform more experiments with different possible combinations of comparison instructions. In this way, it is easy to conclude the optimum method. In addition, we would also like to validate our new experiments with more case studies to make sure the effectiveness of the new methods.

\section{REFERENCES}

[1] Asghari SA, Abdi A, Taheri H, Pedram H, Pourmozaffari S. SEDSR: soft error detection using software redundancy. Journal of Software Engineering and Applications 2012;5(09):664-70.

[2] Jagannathan S, Diggins Z, Mahatme N, Loveless TD, Bhuva BL, Wen SJ et al. Temperature dependence of soft error rate in flip-flop designs: In: IEEE Reliability Physics Symposium (IRPS) 2012.

[3] Baffreau S, Bendhia S, Ramdani M, Sicard E. Characterisation of microcontroller susceptibility to radio frequency interference: In: Fourth IEEE International Caracas Conference on Devices, Circuits and Systems, Oranjestad, Aruba, Netherlands 2002.

[4] Oh N, Mitra S, McCluskey EJ. ED 4 I: error detection by diverse data and duplicated instructions. IEEE Transactions on Computers 2002;51(2):180-99.

[5] Chielle E, Kastensmidt FL, Cuenca-Asensi S. Overhead Reduction in Data-Flow Software-Based Fault Tolerance Techniques: In: FPGAs and Parallel Architectures for Aerospace Applications: Springer 2016, p. 279-91.

[6] Guthaus MR, Ringenberg JS, Ernst D, Austin TM, Mudge T, Brown RB. MiBench: A free, commercially representative embedded benchmark suite: In: Fourth Annual IEEE International Workshop on Workload Characterization, Austin, TX, USA, 2 Dec 2001.

[7] Arasteh B, Bouyer A, Pirahesh S. An efficient vulnerabilitydriven method for hardening a program against soft-error using genetic algorithm. Computers \& Electrical Engineering 2015;48:25-43.

[8] Vankeirsbilck J, Penneman N, Hallez H, Boydens J. Random Additive Signature Monitoring for Control Flow Error Detection. IEEE Transactions on Reliability 2017;66(4):1178-92.

[9] Thati VB, Vankeirsbilck J, Boydens J. Comparative study on data error detection techniques in embedded systems: In: XXV International Scientific Conference 2016.

[10] Reis GA, Chang J, Vachharajani N, Rangan R, August DI. SWIFT: Software Implemented Fault Tolerance: In: International Symposium on Code Generation and Optimization (CGO) 2005, p. 243-54.

[11] Thati VB, Vankeirsbilck J, Penneman N, Pissoort D, Boydens J (eds.). "Comparison of Data Flow Error Detection Techniques in Embedded Systems: an Experimental Study," In press, acepted at ARES 2018.

[12] Cortex -m3 processor: ARM; Available from: https://developer.arm.com/products/processors/cortex$\mathrm{m} /$ cortex-m3.

[13] Heinig A, Korb I, Schmoll F, Marwedel P, Engel M. Fast and Low-Cost Instruction-Aware Fault Injection: In: GIJahrestagung 2013. 